\title{
Thermodynamic Study of Racemic Ibuprofen Separation by Liquid Chromatography Using Cellulose-Based Stationary Phase
}

\author{
Wilson M. Ferrari, Ana C. Nascimento, Jean V. Moreira, and Marco A. Cremasco \\ Chemical Engineering School, University of Campinas (UNICAMP), Av. Albert Einstein 500, Zip Code 13083-852, Campinas, SP, Brazil \\ Correspondence should be addressed to Wilson M. Ferrari; wmcferrari@gmail.com
}

Received 28 April 2016; Revised 12 August 2016; Accepted 25 August 2016

Academic Editor: Masami Shibukawa

Copyright ( 2016 Wilson M. Ferrari et al. This is an open access article distributed under the Creative Commons Attribution License, which permits unrestricted use, distribution, and reproduction in any medium, provided the original work is properly cited.

\begin{abstract}
Ibuprofen is a nonsteroidal anti-inflammatory drug (NSAID), also known for its significant antipyretic and analgesic properties. This chiral drug is commercialized in racemic form; however, only S-(+)-ibuprofen has clinical activities. In this paper the effect of temperature change (from 288.15 to $308.15 \mathrm{~K})$ on the ibuprofen resolution was studied. A column $(250 \times 4.6 \mathrm{~mm})$ packed with tris(3,5-dimethylphenylcarbamate) was used to obtain the thermodynamic parameters, such as enthalpy change $(\Delta H)$, entropy change $(\Delta S)$, variation enthalpy change $(\Delta \Delta H)$, variation entropy change $(\Delta \Delta S)$, and isoenantioselective temperature $\left(T_{\text {iso }}\right)$. The mobile phase was a combination of hexane (99\%), isopropyl alcohol (1\%), and TFA (0.1\%), as an additive. The conditions led to a selectivity of 1.20 and resolution of 4.55 . The first peak, R-(-)-ibuprofen, presented an enthalpy change of $7.21 \mathrm{~kJ} / \mathrm{mol}$ and entropy change of $42.88 \mathrm{~kJ} / \mathrm{K} \cdot \mathrm{mol}$; the last peak, S-(+)-ibuprofen, has an enthalpy change of $8.76 \mathrm{~kJ} / \mathrm{mol}$ and $49.40 \mathrm{~kJ} / \mathrm{K} \cdot \mathrm{mol}$ of entropy change.
\end{abstract}

\section{Introduction}

The importance of stereochemical compound has been studied since Louis Pasteur observed the chiral phenomenon in 1848. Chiral recognition and enantiomer distinction are fundamental phenomena in nature and chemical systems. They are present in several fields, in particular bioactive compounds, drugs, pollutants, agrochemicals, food additives, and flavors. The most significant developments in chirotechnologies were spurred by demands of drug discovery in pharmaceutical industries. The liquid chromatography has been used to provide support in drug discovery, analytical methods, and advances in preparative technique of purification $[1,2]$.

(RS)-2-(4-(2-methylpropyl)phenyl)propanoic acid, also known as ibuprofen, is one of the most widely used nonsteroidal anti-inflammatory drugs (NSAIDs) for the treatment of arthropathies. This enantiomer is a prostaglandin and thromboxane inhibitor [3]. While most currently marketed ibuprofen products are in the form of racemate, it has long been recognized that the two enantiomers have different pharmaceutical activities [4-6]. The ibuprofen structure is shown in Figure 1.
The fundamental basis for distinction of enantiomers (biological or chromatographic system) is the transformation of enantiomers to diastereomers or creation of a diastereomeric relationship between ligated enantiomers (selectand, SA) and a receptor (chiral selector, CS). Equilibrium process such as the CS-SA complexation can be explained by energy balance of free and complexed state and is often studied based on thermodynamic considerations. Thermodynamic quantities of chromatographic equilibrium processes such as the direct enantiomer separation with chiral stationary phase (it uses adsorbents with a chiral selector) can be deduced by measurement of the chromatographic parameters over a certain temperature range from van't Hoff $[1,7-9]$.

This paper aims to study the adsorption of ibuprofen on cellulose tris(3,5-dimethylphenylcarbamate) and to evaluate the effect of temperature in chiral separation. The following parameters were analyzed: enthalpy change $(\Delta H)$, entropy change $(\Delta S)$, and isoenantioselective temperature $\left(T_{\text {iso }}\right)$.

\section{Material and Methods}

2.1. Reagents. Racemic ibuprofen (molar mass $206.29 \mathrm{~g} / \mathrm{mol}$ and purity 98\%) and 1,3,5-tri-tert-butylbenzene (TTBB, 
<smiles>CC(C)Cc1ccc(C(C)C(=O)O)cc1</smiles>

FIGURE 1: Ibuprofen molecular structure.

molar mass $246.44 \mathrm{~g} / \mathrm{mol}$ and purity 97\%) were purchased from Sigma-Aldrich. The chiral analytical column $(250 \mathrm{~mm}$ $\times 4.6 \mathrm{~mm}$ ) was purchased from Phenomenex and originally packed with silica $(5 \mu \mathrm{m})$ coated with cellulose tris $(3,5-$ dimethylphenylcarbamate).

2.2. Instrumentation. The experiments were carried out in a HPLC system; it consisted of a system controller (model CBM-20A), an UV-vis detector (SPD-20A), and two pumps (LC-10AD). The components were originally purchased from Shimadzu, Japan. The column and mobile phase were temperature-controlled using a Quimis Q214M2 (Brazil) circulating water bath. The elution order was determined by a polarimeter model P1010 (Japan).

2.3. Thermodynamic Parameters. The resolution of racemate was studied by means of thermodynamic parameters. Van't Hoff approach was used to obtain the enthalpy change $(\Delta H)$ and entropy change $(\Delta S)$. A similar approach was also used to calculate the difference of enthalpy change $(\Delta \Delta H)$ and entropy change $(\Delta \Delta S)$ for the racemic ibuprofen, respectively. These values are important to obtain the isoenantioselective temperature $\left(T_{\text {iso }}\right)$. The determination of these parameters can be based on the chromatographic parameters, such as the retention factor $\left(k_{i}\right)$ and the selectivity $(\alpha)$. To obtain the isoenantioselective temperature it is necessary to know the porosity $(\varepsilon)$, which was determined by moment analysis [10].

2.4. Chromatographic Parameters. The retention factor is the relation between the number of analytes in stationary phase and the molecule numbers in mobile phase. This parameter is written in terms of analyte retention time $\left(t_{R i}\right)$ and column dead time $\left(t_{0}\right)$ as presented in [11]

$$
k_{i}=\frac{t_{R i}-t_{0}}{t_{0}}=\phi K_{D, i}
$$

where $\phi$ represents the volume phase ratio between the volume of stationary phase and mobile phase, also equal to $\left(1-\varepsilon_{t}\right) / \varepsilon_{t}$.

The selectivity $(\alpha)$ of separation of two components is determined by dividing their retention factors:

$$
\alpha=\frac{k_{j}}{k_{i}}=\frac{t_{R j}-t_{0}}{t_{R i}-t_{0}} .
$$

Chromatography resolution $\left(R_{S}\right)$ and number of plates $\left(N_{i}\right)$ are other important parameters to establish the separation condition:

$$
\begin{aligned}
& R_{s}=\frac{\sqrt{N_{i}}}{4}\left(\frac{k_{i}}{k_{i}+1}\right)\left(\frac{\alpha-1}{\alpha}\right)=1.177 \frac{t_{R j}-t_{R i}}{w_{i}+w_{j}} \\
& N_{i}=5.545\left(\frac{t_{R i}}{w_{i}}\right)^{2},
\end{aligned}
$$

where $w_{i}$ is the peak width at half-height [12].

2.5. Effect of Temperature. Equation (4) describes the linear relation between natural logarithm of the retention factor and inverse of the temperature, in which slope provides enthalpy change and the intercept supplies entropy change. This is modified Van't Hoff equation where $K_{D, i}$ from the original equation was substituted for $k_{i}$. The parameters $\Delta \Delta H$ and $\Delta \Delta S$ can be obtained analogously for (4), as shown in (5). These parameters represent the difference between the less and the more retained compounds, " $i$ " and " $j$ "; respectively, [13-15],

$$
\begin{aligned}
& \ln k_{i}=-\frac{1}{T} \frac{\Delta H_{i}^{0}}{R}+\frac{\Delta S_{i}^{0}}{R}+\ln \frac{1-\varepsilon}{\varepsilon} \\
& \ln \alpha=-\frac{1}{T} \frac{\Delta \Delta H_{j, i}^{0}}{R}+\frac{\Delta \Delta S_{j, i}^{0}}{R},
\end{aligned}
$$

where $T$ is temperature and $R$ is gas constant.

The last term of (4) is related to the physical properties of the stationary phase; thus, it can be considered as a constant since its limitations are not exceeded. If one simplifies the first absolute moment, the total porosity can be obtained experimentally. Therefore, total porosity is determined by (6), in which $v$ is superficial velocity and $L$ is bed length $[10,14,15]$

$$
t_{R}=\frac{L}{v} \varepsilon
$$

Isoenantioselective temperature $\left(T_{\text {iso }}\right)$ is the condition in which the separation does not occur due to both compounds' coelute. If the condition $\alpha=1$ is applied in (5), it is possible to write (7) as follows:

$$
T_{\text {iso }}=\frac{\Delta \Delta H_{j, i}^{0}}{\Delta \Delta S_{j, i}^{0}} .
$$

\section{Results and Discussion}

3.1. Resolution Conditions and Elution Order. The resolution conditions were based on [16-18], at which cellulose tris(3,5dimethylphenylcarbamate) is the chiral selector. Thus, it was decided to use a longer column $(250 \mathrm{~mm} \times 4.6 \mathrm{~mm})$ packed with smaller particles $(5 \mu \mathrm{m})$. Both parameters have contributed to increasing efficiency.

Some mobile phases were tested to resolve the racemic mixture, but only the mobile phase based on hexane promoted satisfactory results. The mobile phase composed of 
TABLE 1: Chromatographic parameters of racemic ibuprofen separation at $308.15 \mathrm{~K}$ : column $(250 \mathrm{~mm} \times 4.6 \mathrm{~mm})$ packed with cellulose tris(3,5-dimethylphenylcarbamate).

\begin{tabular}{lcccc}
\hline Mobile phase & $\alpha$ & $R_{S}$ & $N_{i}$ & $N_{j}$ \\
\hline Hexane/isopropyl alcohol (99/1) & 1.20 & 4.55 & 12976 & 12355 \\
\hline
\end{tabular}
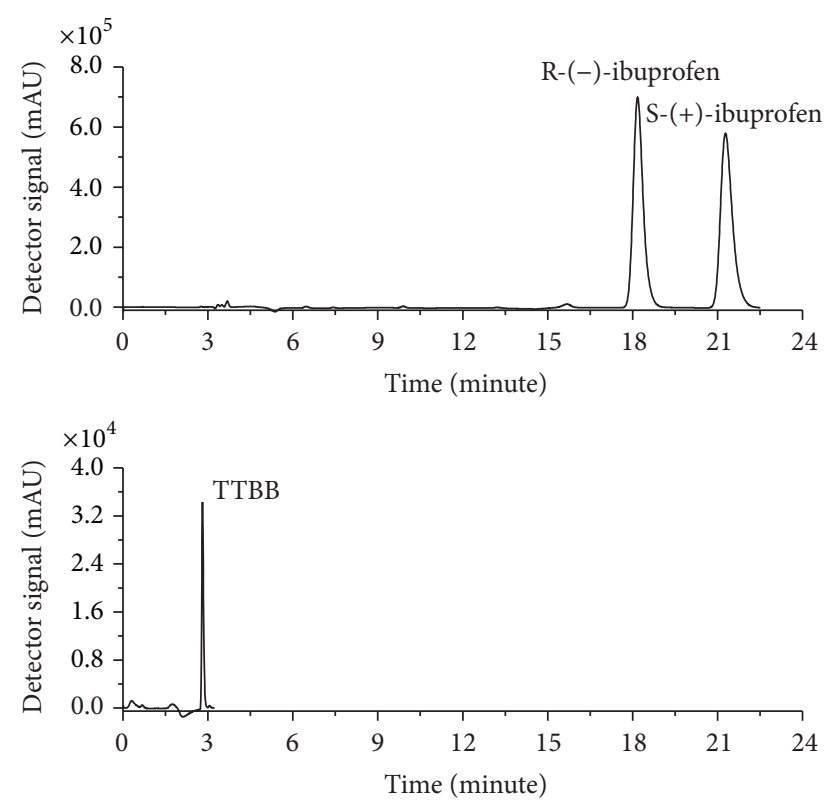

FIGURE 2: Chromatograms of ibuprofen enantiomeric resolution at $308.15 \mathrm{~K}$ and $0.5 \mathrm{~g} / \mathrm{mL}$ and TTBB. Flow ratio: $1 \mathrm{~mL} / \mathrm{min}$; wavelength: $220 \mathrm{~nm}$ for ibuprofen and $254 \mathrm{~nm}$ for TTBB; mobile phase: hexane/isopropyl alcohol (99/1).

hexane and isopropyl alcohol, in the proportion 99/1, was chosen for chiral resolution. Trifluoroacetic acid (TFA) was also used as an additive; its proportion on mobile phase was $0.1 \%$, in volumetric base. All the conditions set for the chromatographic resolution provided values of $\alpha$ and $N_{i}$ above minimum levels required for effective chromatographic resolution $\left(\alpha>1.20, R_{S}>1.50\right.$, and $\left.N_{i}>2000\right)$. Table 1 summarizes all the mentioned values [19].

After setting the separation conditions, the elution order was defined through a polarimetric technique. For this, the HPLC was used in batch mode to resolve the racemic mixture in R-ibuprofen and S-ibuprofen. The rotary evaporator reduced each sample for the polarimeter sample cell volume. A calibration curve provided the concentration of both samples: $0.4 \mathrm{~g} / \mathrm{L}$ for S-ibuprofen and $1.11 \mathrm{~g} / \mathrm{L}$ for R-ibuprofen. Finally, the polarimeter identified the less and more retained compounds as (-)-ibuprofen and (+)-ibuprofen, respectively. Thus, in Figure 2, the first peak is R-(-)-ibuprofen while the second peak is S-(+)-ibuprofen.

3.2. Total Porosity. Porosity was calculated from the first moment method (6). This method requires a tracer compound small enough to pass through both particle and bed voids. TTBB was used to determine the porosity of chiral

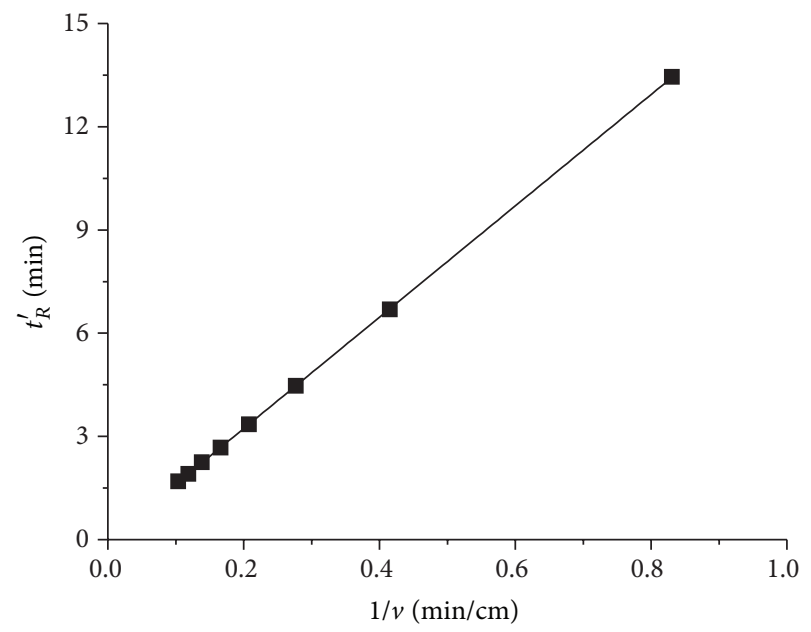

FIGURE 3: Total porosity.

TABLE 2: Retention factor obtained in racemic ibuprofen separation.

\begin{tabular}{cccc}
\hline$T(\mathrm{~K})$ & $k_{i}$ & $k_{j}$ & $\alpha$ \\
\hline 288.15 & 4.72 & 5.43 & 1.15 \\
293.15 & 4.88 & 5.68 & 1.16 \\
298.15 & 5.11 & 5.96 & 1.17 \\
303.15 & 5.38 & 6.37 & 1.18 \\
308.15 & 5.74 & 6.90 & 1.20 \\
\hline
\end{tabular}

columns packed with cellulose tris(3,5-dimethylphenylcarbamate). The flow rate was changed from 0.2 up to $1.6 \mathrm{~mL} / \mathrm{min}$ with a $0.2 \mathrm{~mL} / \mathrm{min}$ step. The results are shown in Figure 3.

The slope presented in Figure 3 provides the value of 0.647 for porosity.

3.3. Thermodynamic Parameters. The adsorption phenomenon of the enantiomeric compounds is macroscopic thermodynamic quantities, which does not consider the surface heterogeneity of the stationary phase and the associated distinct adsorption behavior of enantiomers at different sites. Under the circumstances, Van't Hoff analysis is useful to determine global information about the phenomenon [1].

Using (4), modified Van't Hoff equation, one can obtain the thermodynamic parameters. The temperatures chosen were 288.15, 293.15, 298.15, 303.15, and 308.15 K. At each temperature, racemic ibuprofen samples were injected at $0.5 \mathrm{~g} / \mathrm{L}$. Figure 4 and Table 2 present a summary of chromatograms and chromatographic values obtained from these resolutions.

In Figure 4, an increase in retention time value was observed as temperature rises. This behavior has been reported in literature; however, it is not the most common one [1]. This condition will be discussed later in this paper.

The retention factors from Table 2 were used to plot Figure 5. From this figure and utilizing (4), it was possible to obtain $\Delta H$ and $\Delta S$, for ibuprofen enantiomers as presented in Table 3. The errors for enthalpy and entropy change were, respectively, $6 \%$ and $9 \%$. 


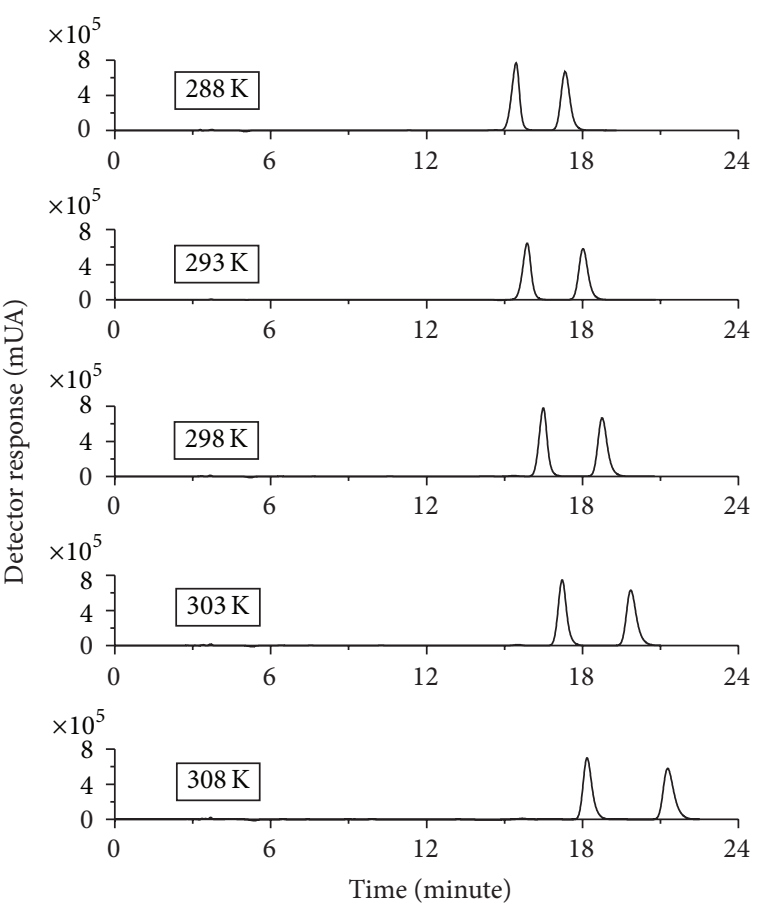

Figure 4: Chromatograms of the racemic ibuprofen at several temperatures. Flow rate: $1 \mathrm{~mL} / \mathrm{min}$.

TABLE 3: Thermodynamic parameters of racemic ibuprofen adsorbed on cellulose tris(3,5-dimethylphenylcarbamate).

\begin{tabular}{lccc}
\hline Compound & $\Delta H(\mathrm{~kJ} / \mathrm{mol})$ & $\Delta S(\mathrm{~kJ} / \mathrm{K} \cdot \mathrm{mol})$ & $R^{2}$ \\
\hline R-(-)-ibuprofen & 7.21 & 42.88 & 0.975 \\
S-(+)-ibuprofen & 8.76 & 49.40 & 0.972 \\
\hline
\end{tabular}

Both relations presented in Figure 4 are linear, suggesting that multiple mechanisms of retention do not occur $[19,20]$. Furthermore, $\Delta H$ of compounds is lower than $50 \mathrm{~kJ} / \mathrm{mol}$ demonstrating physical adsorption [11]. The interactions of physisorption are characterized by relatively weak bonds. Otherwise, it would be necessary to modify the chromatographic system to elute analytes.

According to [1] the adsorption phenomenon in liquid chromatographic enantiomer resolution is driven by (mostly electrostatic type) noncovalent interactions. Thermodynamic quantities have shown negative heats of adsorption for a wide variety of different mobile phase, stationary phase, and analyte. Additionally, there is a decrease in retention time as temperature rises; this occurs due to exothermic characteristic of adsorption as [1] also mentioned that in most of cases the compensation effect (also called enthalpy-entropy compensation) occurs. However, both enthalpy changes from Table 3 presented positive values indicating an endothermic phenomenon. Furthermore, the retention times in Figure 4 increase together as temperature rises. This aspect can be linked with a favorable entropy contribution. This indicates that when the analyte is bound to the stationary phase, the solvent molecules overload the adsorption site favoring the entropy increase.

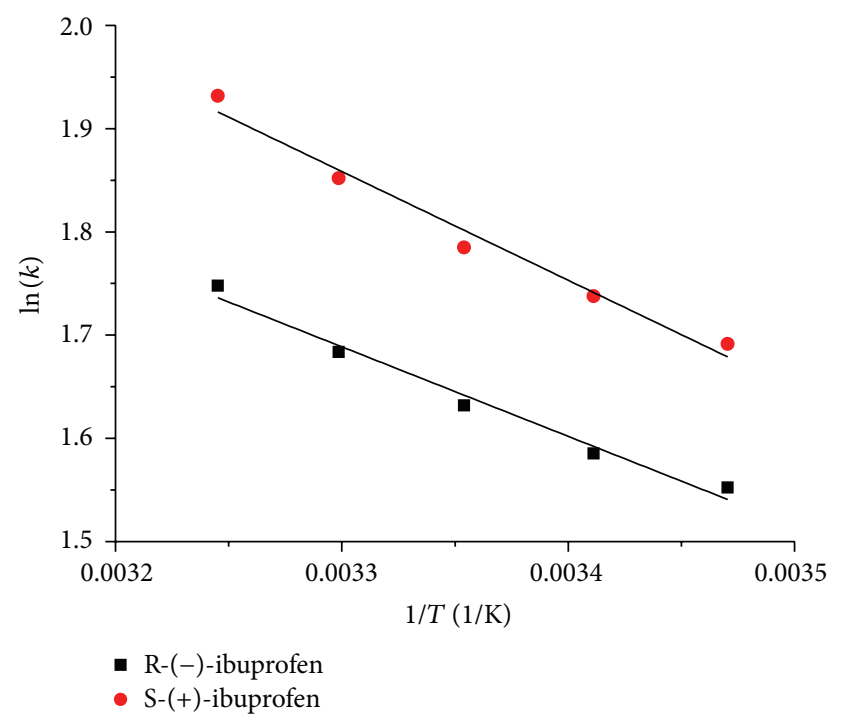

FIgURE 5: Van't Hoff plots for the determination of $\Delta H$ and $\Delta S$.

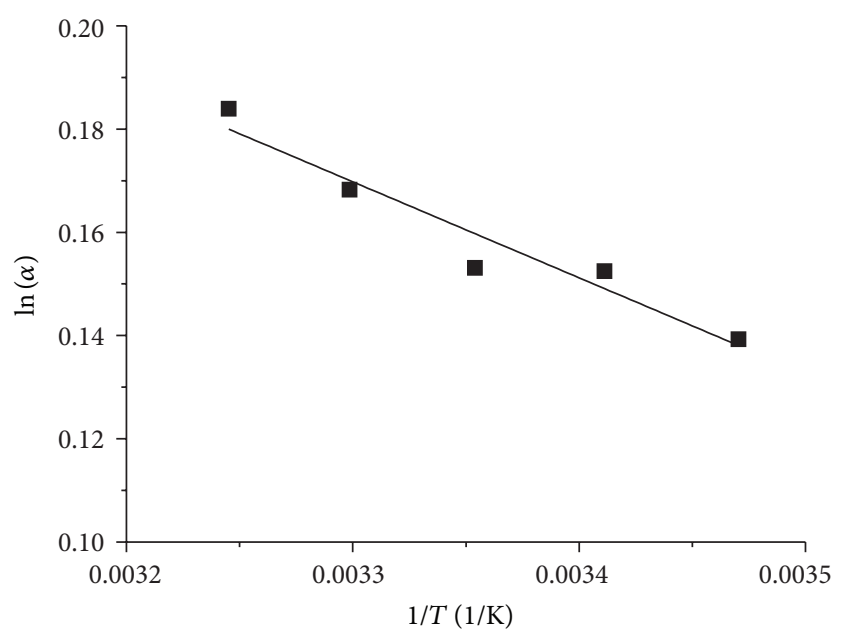

FIGURE 6: Linearization for determining of $\Delta \Delta H$ and $\Delta \Delta S$.

The selectivity from Table 2 was also employed to analyze the separation between R-(-)-ibuprofen and S-(+)-ibuprofen. Parameters $\Delta \Delta H$ and $\Delta \Delta S$ were calculated by means of (5). The linearization proposal for this equation is presented in Figure 6.

The linearization of $\ln (\alpha)$ versus $1 / T$ provided values of $1.55 \mathrm{~kJ} / \mathrm{mol}$ and $6.52 \mathrm{~J} /(\mathrm{mol} \cdot \mathrm{K})$ for $\Delta \Delta H$ and $\Delta \Delta S$, respectively, and it also presented a $s$-squared of 0.915 . Error for $\Delta \Delta H$ was $12 \%$ and error for $\Delta \Delta S$ was $15 \%$. Equation (7) provided $237.38 \mathrm{~K}$ for $T_{\text {iso }}$.

Thermodynamic parameters are global information about the adsorption mechanism in a chromatographic column. The adsorption phenomenon involved in this process depends on solute type, mobile phase, and stationary phase [1]. For enantiomeric separation the adsorption mechanism is related to the chiral recognition. According to information from Figure 6, the adsorption is controlled by entropy $(|T \cdot \Delta \Delta S|>|\Delta \Delta H|)$ because entropy contribution is more 
relevant than enthalpy contribution for the ibuprofen retention mechanism in cellulose tris(3,5-dimethylphenylcarbamate).

However, the separation will not be driven by entropy for all temperature ranges; this happens due to $T_{\text {iso }}$; it is a boundary temperature for driven forces in this case. This becomes evident when the conditions for $T_{\text {iso }}$ are applied in GibbsHelmholtz equation and the result is zero for energy change $(\Delta G)$. For this case, adsorption phenomenon at temperatures below $T_{\text {iso }}, 237.38 \mathrm{~K}$, will be driven by entropy; on the other hand, temperatures above $T_{\text {iso }}$ will be driven by enthalpy. It is common that the adsorption phenomenon is exothermic and it occurs above $T_{\text {iso }}[18,20-23]$. Probably the elution order of compounds could be inverted in temperature lower than $T_{\text {iso }}$. Moreover, the phenomenon would be controlled by enthalpy change, the selectivity would decrease as temperature rises, and the adsorption would be exothermic if ibuprofen were separated below $T_{\text {iso }}[20,22,23]$.

\section{Conclusions}

Racemic ibuprofen can be separated using a chiral stationary phase based on cellulose tris(3,5-dimethylphenylcarbamate) and a high nonpolar mobile phase. The mobile phase composed of hexane (99\%) and isopropyl alcohol (1\%) was chosen to separate the mixture. Trichloroacetic acid at $0.1 \%$ was employed as an additive; on this configuration of mobile phase and stationary phase, the S-(+)-ibuprofen is the most retained enantiomer.

Thermodynamic parameters obtained from Figure 4 demonstrated that the adsorption is physical and the separation phenomenon is endothermic. It shows that high temperatures favored the separation. Furthermore, the parameters from Figure 5 show that phenomenon of separation is controlled by entropy $(|T \cdot \Delta \Delta S|>|\Delta \Delta H|)$ at temperatures above $T_{\text {iso }}$.

\section{Nomenclature}

\section{Symbols}

$\Delta \Delta H$ : Variation of enthalpy change between more and less retained compound $(\mathrm{J} / \mathrm{mol})$

$\Delta \Delta S$ : Variation of entropy change between more and less retained compound $(\mathrm{J} / \mathrm{K} \cdot \mathrm{mol})$

$\Delta H: \quad$ Standard enthalpy change $(\mathrm{J} / \mathrm{mol})$

$\Delta G: \quad$ Standard Gibbs free energy change $(\mathrm{J} / \mathrm{mol})$

$\Delta S: \quad$ Standard entropy change $(\mathrm{J} / \mathrm{K} \cdot \mathrm{mol})$

$k_{i}$ : $\quad$ Retention factor of the least retained compound

$k_{j}$ : $\quad$ Retention factor of the most retained compound

$L: \quad$ Column length $(\mathrm{cm})$

$N_{i}$ : Number of plates of the least retained compound

$N_{j}$ : Number of plates of the most retained compound

$R: \quad$ Universal gas constant $(8.3144 \mathrm{~J} / \mathrm{mol} \cdot \mathrm{K})$

$R_{S}$ : Resolution
T: $\quad$ Absolute temperature (K)

$t_{0}:$ Column dead time ( $\left.\mathrm{min}\right)$

$T_{\text {iso }}$ : Isoenantioselective temperature (K)

$t_{R i}$ : Retention time of the least retained compound plus dead time ( $\mathrm{min}$ )

$t_{R j}$ : Retention time of the most retained compound plus dead time (min)

$w_{i}$ : Peak width at half-height of the least retained compound

$w_{j}$ : Peak width at half-height of the most retained compound.

\section{Greek Letters}

$\alpha$ : Selectivity

$\varepsilon$ : Porosity

$\nu$ : Superficial velocity $(\mathrm{cm} / \mathrm{min})$.

\section{Competing Interests}

The authors declare that there is no conflict of interests regarding the publication of this paper.

\section{Acknowledgments}

The financial support of this research by $\mathrm{CNPq}$ (Brazil) is gratefully acknowledged.

\section{References}

[1] M. Lämmerhofer, "Chiral recognition by enantioselective liquid chromatography: mechanisms and modern chiral stationary phases," Journal of Chromatography A, vol. 1217, no. 6, pp. 814856, 2010.

[2] I. Ali, S. D. Alam, Z. A. Al-Othman, and J. A. Farooqi, "Recent advances in SPE-chiral-HPLC methods for enantiomeric separation of chiral drugs in biological samples," Journal of Chromatographic Science, vol. 51, no. 7, pp. 645-654, 2013.

[3] J. S. Yoon, D.-C. Jeong, J.-W. Oh et al., "The effects and safety of dexibuprofen compared with ibuprofen in febrile children caused by upper respiratory tract infection," British Journal of Clinical Pharmacology, vol. 66, no. 6, pp. 854-860, 2008.

[4] M. Venu Madhav and C. B. Ching, "Study on the enzymatic hydrolysis of racemic methyl ibuprofen ester," Journal of Chemical Technology and Biotechnology, vol. 76, no. 9, pp. 941-948, 2001.

[5] M. Yousefi, M. Mohammadi, and Z. Habibi, "Enantioselective resolution of racemic ibuprofen esters using different lipases immobilized on octyl sepharose," Journal of Molecular Catalysis B: Enzymatic, vol. 104, pp. 87-94, 2014.

[6] H. Li, X. Jiang, W. Xu, Y. Chen, W. Yu, and J. Xu, "Numerical determination of non-Langmuirian adsorption isotherms of ibuprofen enantiomers on Chiralcel OD column using ultraviolet-circular dichroism dual detector," Journal of Chromatography A, vol. 1435, pp. 92-99, 2016.

[7] Z. Pataj, I. Ilisz, A. Aranyi et al., "LC separation of $\gamma$-amino acid enantiomers," Chromatographia, vol. 71, no. 1, pp. S13-S19, 2010.

[8] A. Cavazzini, L. Pasti, A. Massi, N. Marchetti, and F. Dondi, "Recent applications in chiral high performance liquid chromatography: a review," Analytica Chimica Acta, vol. 706, no. 2, pp. 205-222, 2011. 
[9] I. Ilisz, N. Grecsó, M. Palkó, F. Fülöp, W. Lindner, and A. Péter, "Structural and temperature effects on enantiomer separations of bicyclo[2.2.2] octane-based 3-amino-2-carboxylic acids on cinchona alkaloid-based zwitterionic chiral stationary phases," Journal of Pharmaceutical and Biomedical Analysis, vol. 98, pp. 130-139, 2014.

[10] M. A. Cremasco, B. J. Hritzko, Y. Xie, and N. H. L. Wang, "Parameters estimation for amino acids adsorption in a fixed bed by moment analysis," Brazilian Journal of Chemical Engineering, vol. 18, no. 2, pp. 181-194, 2001.

[11] A. Seidel-Morgenstern, M. Schulte, and A. Epping, "Fundamentals and general terminology," in Preparative Chromatography, pp. 7-46, 2nd edition, 2012.

[12] C. H. Collins, G. L. Braga, and P. S. Bonato, Fundamentos de Cromatografia, Unicamp, 2006.

[13] A. L. Myers and J. M. Prausnitz, "Thermodynamics of mixedgas adsorption," AIChE Journal, vol. 11, no. 1, pp. 121-127, 1965.

[14] D. M. Ruthven, Principles of Adsorption and Adsorption Processes, John Wiley \& Sons, 1984.

[15] G. Guiochon, A. Felinger, and D. G. Shirazi, Fundamentals of Preparative and Nonlinear Chromatography, Academic Press, 2006.

[16] A. Ducret, M. Trani, P. Pepin, and R. Lortie, "Comparison of two HPLC techniques for monitoring enantioselective reactions for the resolution of (R,S)-ibuprofen: chiral HPLC vs achiral HPLC linked to an optical rotation detector," Biotechnology Techniques, vol. 9, no. 8, pp. 591-596, 1995.

[17] P. S. Bonato, M. P. F. M. Del Lama, and R. de Carvalho, "Enantioselective determination of ibuprofen in plasma by highperformance liquid chromatography-electrospray mass spectrometry," Journal of Chromatography B: Analytical Technologies in the Biomedical and Life Sciences, vol. 796, no. 2, pp. 413-420, 2003.

[18] W. M. Ferrari and M. A. Cremasco, "Thermodynamic study of the separation of racemic ibuprofen by chiral liquid chromatograph," in Anais do XX Congresso Brasileiro de Engenharia Química-COBEQ 2014, vol. 1, Blucher, São Paulo, Brazil, 2015.

[19] V. R. Meyer, Practical High-Performance Liquid Chromatography, John Wiley \& Sons, 2013.

[20] T. D. Booth and I. W. Wainer, "Mechanistic investigation into the enantioselective separation of mexiletine and related compounds, chromatographed on an amylose tris(3,5-dimethylphenylcarbamate) chiral stationary phase," Journal of Chromatography A, vol. 741, no. 2, pp. 205-211, 1996.

[21] C. B. Castells and P. W. Carr, "A study of the thermodynamics and influence of temperature on chiral high-performance liquid chromatographic separations using cellulose tris $(3,5-$ dimethylphenylcarbamate) coated zirconia stationary phases," Chromatographia, vol. 52, no. 9-10, pp. 535-542, 2000.

[22] K. Fulde and A. W. Frahm, "Temperature-induced inversion of elution order in the enantioseparation of sotalol on a cellobiohydrolase I-based stationary phase," Journal of Chromatography A, vol. 858, no. 1, pp. 33-43, 1999.

[23] B. Yao, F. Zhan, G. Yu et al., "Temperature-induced inversion of elution order in the chromatographic enantioseparation of $1,1^{\prime}$ bi-2-naphthol on an immobilized polysaccharide-based chiral stationary phase," Journal of Chromatography A, vol. 1216, no. 28, pp. 5429-5435, 2009. 

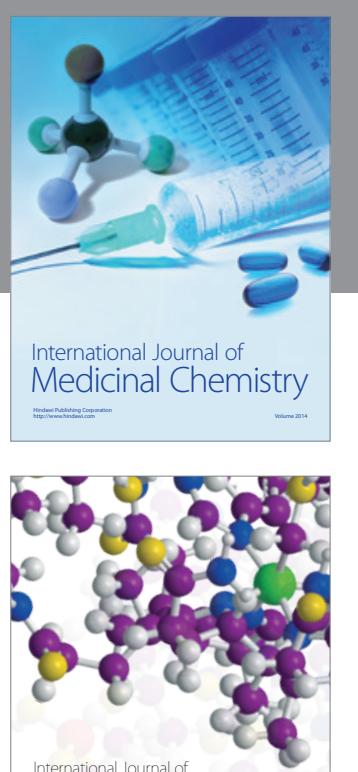

Carbohydrate Chemistry

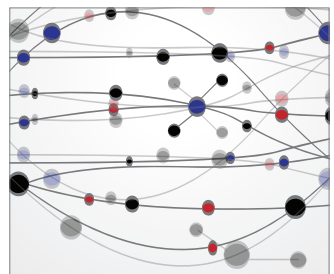

The Scientific World Journal
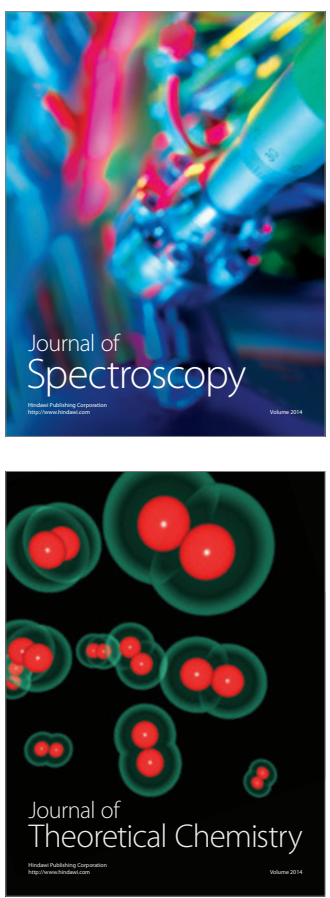
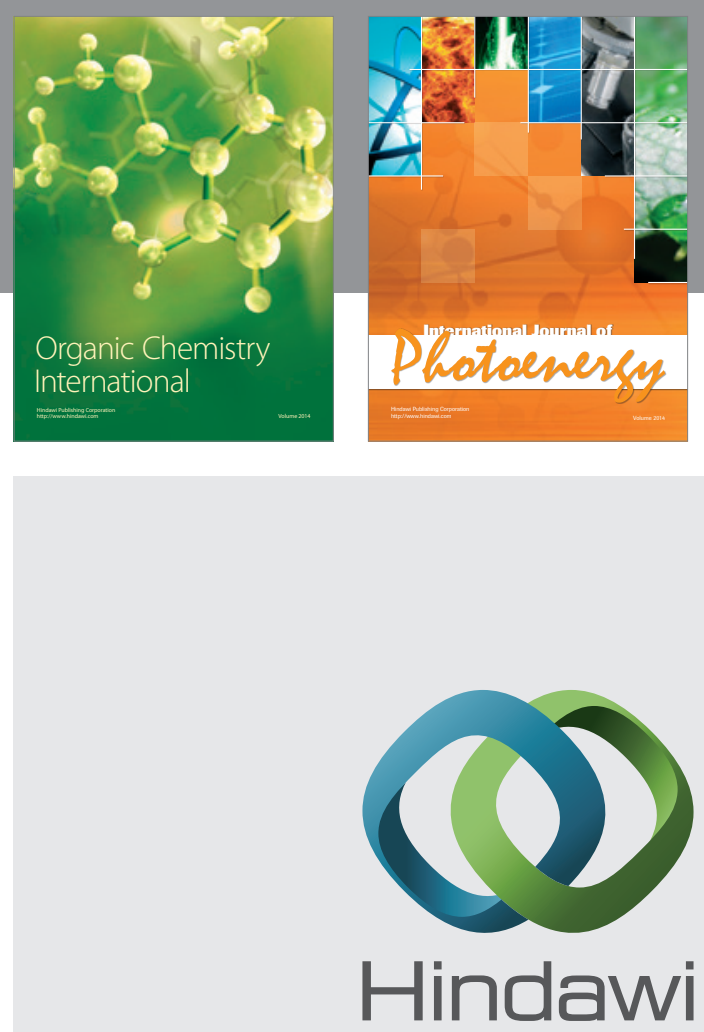

Submit your manuscripts at

http://www.hindawi.com

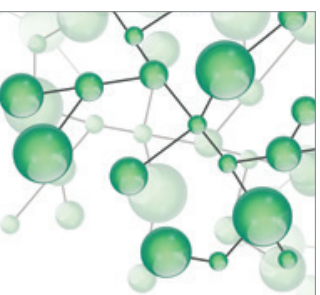

International Journal of

Inorganic Chemistry

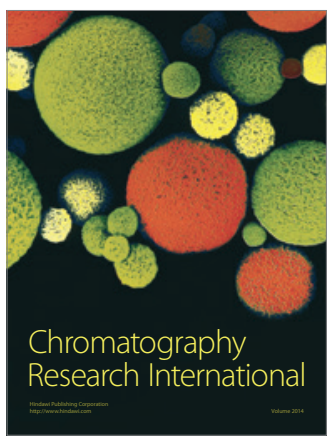

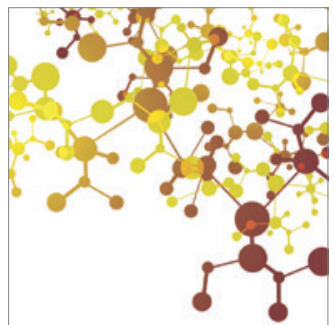

Applied Chemistry
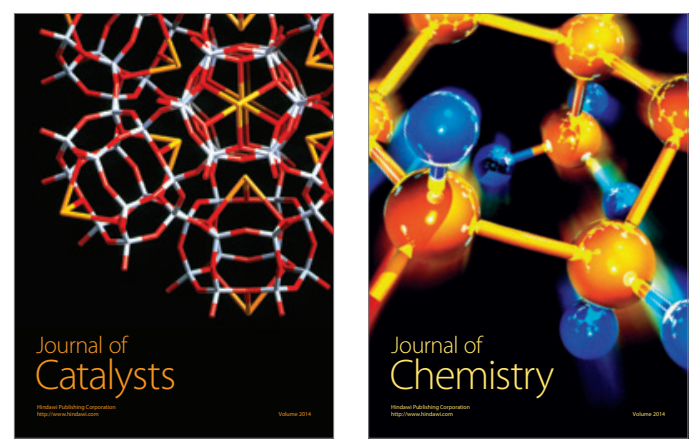
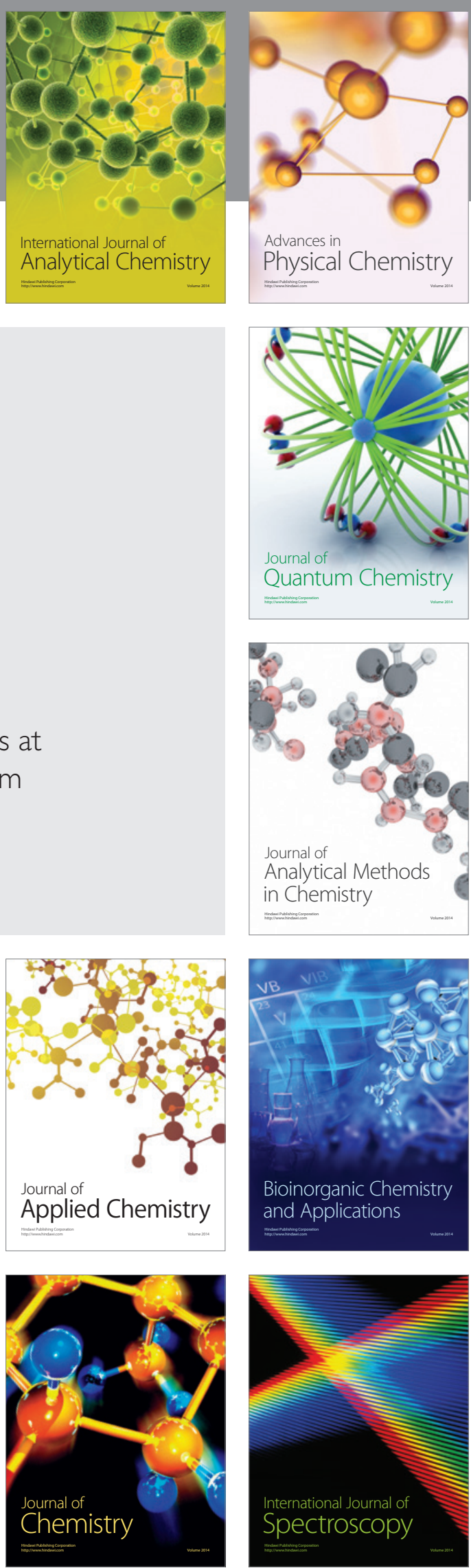